\title{
Diversity and Characterization of Larval Habitats of Anopheles Mosquitoes in Urban Areas in Benin: Influence of Physicochemical and Bacteriological Factors on Larval Density
}

\author{
Donald Hessou-Djossou \\ Ecole Normale Superieure de Natitingou \\ Innocent Djègbè ( $\square$ djegbe1@yahoo.fr) \\ Ecole Normale Superieure de Natitingou \\ Koffi Mensah Ahadji-Dabla \\ Universite de Lome Faculte des Sciences \\ Odilon M. Nonfodji \\ Ecole Normale Superieure de Natitingou \\ Geneviève Tchigossou \\ IITA: International Institute of Tropical Agriculture \\ Rousseau Djouaka \\ IITA: International Institute of Tropical Agriculture \\ Sylvie Cornelie \\ IRD: Institut de recherche pour le developpement \\ Luc Djogbenou \\ Universite d'Abomey-Calavi \\ Martin Akogbeto \\ Centre de Recherche Entomologique de Cotonou \\ Fabrice Chandre \\ IRD: Institut de recherche pour le developpement
}

\section{Research Article}

Keywords: Ecology, Physicochemical parameters, Faecal coliforms, Larval density, Anopheles

Posted Date: January 24th, 2022

DOI: https://doi.org/10.21203/rs.3.rs-1262109/v1

License: (c) (i) This work is licensed under a Creative Commons Attribution 4.0 International License. Read Full License 


\section{Abstract}

Background: The implementation of antilarval strategies in the fight against malaria vectors requires fundamental knowledge of their oviposition sites. This study aimed to assess the spatial and temporal distribution of Anopheles breeding sites as well as the influence of abiotic and biotic factors on the proliferation of larvae in urban and non-urban environments in Benin.

Methods: Larval surveys were carried out during the rainy and dry seasons in urbanized and non-urbanized areas in the cities of Parakou, Natitingou, Bohicon and Cotonou in Benin. The Anopheles larval breeding sites were georeferenced and characterized by their nature, typology, physicochemical $(\mathrm{pH}$, temperature, dissolved oxygen, conductivity, turbidity, salinity) and biological attributes (larval density and coliform density).

Results: A total of 198 different types and positive breeding sites for Anopheles larvae were identified. These include 163 (82.3\%) breeding sites during the rainy season and 35 (17.7\%) during the dry season. Out of these habitats, $61.9 \%$ were located in urbanized areas with a predominance of puddles. The Principal Component Analysis (PCA) revealed a high positive correlation of larval density with temperature, dissolved oxygen and salinity in the coastal zone (Cotonou). In addition, the cross-analysis of the microbiological results with the larval density has generally shown a significant negative correlation between the larval productivity of anopheles breeding sites and their faecal coliforms load.

Conclusion: This study showed multiple larval habitats of Anopheles in urban environments due to several anthropogenic factors and larval adaptation to unusual ecosystems.

\section{Background}

In recent years, there has been renewed interest in urban malaria, particularly in sub-Saharan Africa. It is estimated that nearly 150 million city dwellers are currently at risk of malaria and that 6 to $28 \%$ of annual malaria cases in Africa occur in urban areas $[1,2]$. These figures could be revised upwards due to the galloping demographic growth of the continent and the massive migration of populations to urban centres. Indeed, according to recent projections, more than $60 \%$ of the African population will live in urban areas by 2050 [3]. In addition, the rapid and anarchic urbanization process in large cities beyond political power. This urbanization causes the emergence of new lifestyles which lead to an increase in mosquito larval habitats, specifically those of Anopheles species, making these cities a strong reservoir of potential vectors [4].

In Benin, as in most sub-Saharan countries, malaria remains a major public health problem with an increase in malaria incidence and associated mortality which doubled between 2010 and 2017 [5]. Currently, the two main vector control methods recommended by the National Malaria Control Program (NMCP) are based on the use of insecticides and target adult mosquitoes through indoor residual spraying (IRS) $[6,7,8]$, and the use of long-lasting insecticidal nets (LLINs) $[9,10]$. However, the development of resistance of Anopheles to insecticides, a more exophagous behaviour of Anopheles and the existence of residual transmission when people are not protected by IRS or LLINs underline the interest of developing complementary methods [10,11]. Among these, larval control, particularly in urban areas where habitats are limited and easily identifiable, has recently been recommended by the World Health Organization (WHO) [12]. In many countries around the world, larval control through the systematic application of larvicides has been used to complement IRS and LLINs in malaria vector control [13]. Controlling mosquito larval populations is often beneficial because the larvae are usually concentrated, relatively immobile and often easily accessible [14]. Appropriate management of larval habitats could help suppress vector densities and therefore transmission of malaria; but this management requires in-depth and update knowledge of the distribution and typology of the breeding sites colonized by malaria vectors.

Many studies have shown that the establishment of an effective vector control method must include the eco-climatic factors, bioecology of mosquito vector, spatio-temporal distribution and physicochemical characterization of the oviposition sites and the level of sensitivity of the adult mosquito to insecticides $[15,16,17]$. Indeed, various factors influence the density and distribution of these mosquito species and their resistance to insecticides. These concern the climatic factors, plant cover, the breeding sites types and anthropogenic factors [18]. In addition, the physicochemical (temperature, salinity, hydrogen potential, nitrates, etc.) and bacteriological parameters of the breeding sites water can also influence the distribution of culicidal species [16, 19]. Such data on mosquito bioecology is lacking in Benin, which constitutes a serious handicap for evaluation of vector control strategies.

This study aimed to investigate on the distribution of Anopheles larvae as well as the biotic and abiotic habitats characteristics favourable to their development in four cities in Benin. 


\section{Materials And Methods \\ Study areas}

The study was carried out in four cities, selected along a transect from North to South Benin, so as to cover the different ecological

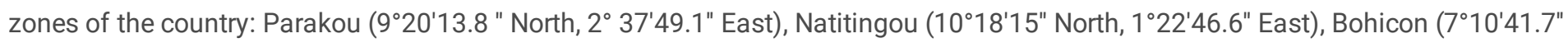
North, $2^{\circ} 4^{\prime} 0.1^{\prime \prime}$ East) and Cotonou (6 $6^{\circ} 21^{\prime} 55.3^{\prime \prime}$ North, $2^{\circ} 25^{\prime} 6^{\prime \prime}$ East). Four districts were selected in each city; two urban and two nonurban (Figure 1).

The city of Parakou in the department of Borgou is at an average altitude of $350 \mathrm{~m}$ above the sea level with a modest relief (i.e., the geographical difference between the highest and lowest elevation point in an area). It covers an area of $441 \mathrm{sq} \mathrm{km}$ with a population of 254,254 inhabitants [20]. In Parakou, the climate is tropical and humid (South-Sudanese climate). It is characterized by an annual alternation of a rainy season (May to October) and a dry season (November to April). The lowest temperatures are recorded in December-January. The average annual rainfall is $1,200 \mathrm{~mm}$, with a maximum occurring between July and September. Manufacturing, commerce and urban agriculture are the main activities in Parakou [21].

Natitingou is located in the department of Atacora in northwestern Benin, and has an altitude of around $500 \mathrm{~m}$ above sea level with a rugged terrain. It covers an area of $3,045 \mathrm{sq} \mathrm{km}$ with a population of 103,843 inhabitants [20]. The climate is Sudano-Guinean, characterized by two seasons: a rainy season (May to October), and a dry season (November to April). The city of Natitingou is heavily watered, with $1,400 \mathrm{~mm}$ of rainfall sometimes recorded per year. The average temperature is around $27^{\circ} \mathrm{C}$ with variations of $17^{\circ} \mathrm{C}$ to $35^{\circ} \mathrm{C}$ during the harmattan. The main activities are agriculture, tourism, crafts and commerce [22].

The city of Bohicon in central Benin in the department of Zou, is characterized by a vegetation made up of trees with tough, worming leaves that resist heat. It covers an area of $44 \mathrm{sq} \mathrm{km}$ with a population of 171,781 inhabitants [20]. Bohicon enjoys a transitional subequatorial climate with two rainy seasons (April to June and September to November) and two dry seasons (July to August and December to March). The rainfall amounts to an average of $1,025 \mathrm{~mm}$ per year and temperature varies between 25 and $34^{\circ} \mathrm{C}$. The main activities of the population are commerce, crafts and agriculture [23].

Cotonou, the economic capital of Benin is located on the coastal strip that stretches between Nokoué Lake and the Atlantic Ocean, made up of alluvial sands with a maximum height of about five meters. The city of Cotonou covers an area of $79 \mathrm{sq} \mathrm{km}$ with a population of $1,228,667$ inhabitants [20]. The climate is equatorial, where two rainy seasons alternate with two dry seasons. The rainfall varies between 900 and $1,200 \mathrm{~mm}$ while the average temperature fluctuates between 18 and $35^{\circ} \mathrm{C}$. The relief of Cotonou is not very rugged with swamps and lowlands very suitable for vegetable gardening. The city is full of a large number of processing and storage factories [24].

\section{Surveys and mapping of anopheles breeding sites}

The larval habitats of anopheles mosquito were surveyed from August to September 2020 in the rainy season and from January to February 2021 in the dry season. During these surveys, water collections to harbour pre-imaginal stages (all stages combined) of mosquitoes were observed. They are considered as breeding sites if the Anopheles immature stages are present. The different breeding sites encountered were characterized by their container, their artificial or natural character, and then georeferenced using a Garmin GPS navigator (GarminInc., Kansas, USA).

Physicochemical parameters such as $\mathrm{pH}$, temperature, conductivity, salinity, TDS, dissolved oxygen and turbidity of water samples from breeding sites were measured in-situ using a HANNA HI 991001 pH meter, a VWR CO300 multiparametric conductivity meter, a WTW OXI 3205 oximeter and a HANNA HI 93703 turbidimeter, respectively. After the measurement, at least $200 \mathrm{~mL}$ of water was taken from each sampling breeding site and placed into a sterile flask. Then, the flask was placed into an icebox and brought back to the laboratory for microbiological analysis.

\section{Larval collection}

Anopheline larvae were collected using the standard dipping techniques following the method described by Silver, 2007 [25]. In each breeding site, 3 to 10 dips were taken depending on the larval habitat size, using a standard dipper $350 \mathrm{~mL}$. After each dip, the contents were transferred in a plastic container and the larval count was estimated [26]. The larvae were then stored in well-labelled plastic 
containers and transported to the Entomology laboratory of Ecole Normale Supérieure de Natitingou for rearing and morphological identification [27].

\section{Microbiological analysis of water from the breeding sites}

The level of microbiological pollution of the breeding sites was evaluated. The isolation of total coliforms and Escherichia coli was performed at $37^{\circ} \mathrm{C}$ while that of faecal coliforms at $44^{\circ} \mathrm{C}$ according to the technique of filtration through a nitrocellulose membrane of $0.45 \mu \mathrm{m}$ porosity on Chromogenic Coliform Agar according to the protocol described by Nonfodji et al [28].

\section{Statistical analysis}

Pearson's chi-square test was used to compare the proportions of larvae in the different habitat types between seasons. Correlations between recorded physicochemical variables and larval densities were assessed using Pearson's correlation analysis and Principal Component Analysis (PCA). All statistical analysis was performed using R software version 4.0.3 (R Development Core Team, 2020).

\section{Results}

\section{Typology and distribution of anopheles larval habitats}

Overall, 198 breeding sites of Anopheles larvae were identified and characterized during surveys in the four cities, including 163 breeding sites (82.3\%) in the rainy season (Figure 2) and 35 breeding sites (17.7\%) in the dry season (Figure 3). A significant difference was observed in the distribution of Anopheles breeding sites according to the seasons $\left(X^{2}=58.093, d f=1, P<0.0001\right)$ (Table 1$)$. During the rainy season, out of 163 breeding sites, 101 (61.9\%) were found in urban districts compared to 62 (38.1\%) in non-urban districts. In the dry season, 20 (57.1\%) and 15 (42.9\%) breeding habitats were found in urban and non-urban districts, respectively. No difference was observed in the distribution of breeding sites between urban and non-urban districts according to the collection period. All the 198 breeding sites were categorized into 17 types including puddles $(45.9 \%, n=91)$, tyres $(14.6 \%, n=29)$ and gutters $(11.1 \%, n=22)$ which had the highest frequencies (Table 2). During the rainy season, puddles were the most frequent habitat types encountered in Natitingou $(40 \%, n=14)$, Bohicon $(67.5 \%, n=27)$ and Cotonou $(77.8 \%, n=35)$ while tyres $(32.6 \%, n=14)$ and puddles $(20.9 \%, n=9)$ were mostly found in Parakou.

Table 1

Distribution of breeding sites according to the level of urbanization of the study sites in rainy and dry seasons

\begin{tabular}{|c|c|c|c|c|c|c|c|c|c|c|}
\hline \multirow[t]{3}{*}{ Cities } & \multicolumn{5}{|c|}{ Rainy Season } & \multicolumn{5}{|c|}{ Dry Season } \\
\hline & \multicolumn{2}{|c|}{ Urban areas } & \multicolumn{2}{|c|}{ Non-urban areas } & \multirow{2}{*}{$\begin{array}{l}\text { Total } \\
\mathbf{N}(\%)\end{array}$} & \multicolumn{2}{|c|}{ Urban areas } & \multicolumn{2}{|c|}{ Non-urban areas } & \multirow{2}{*}{$\begin{array}{l}\text { Total } \\
\mathbf{N}(\%)\end{array}$} \\
\hline & $\mathbf{N}$ & $\%$ & $\mathbf{N}$ & $\%$ & & $\mathrm{n}$ & $\%$ & $\mathbf{N}$ & $\%$ & \\
\hline Parakou & 20 & 46.5 & 23 & 53.5 & $43(100)$ & 3 & 30 & 7 & 70 & $10(100)$ \\
\hline Natitingou & 26 & 74.3 & 9 & 25.7 & 35 (100) & 5 & 100 & 0 & 0 & $5(100)$ \\
\hline Bohicon & 27 & 67.5 & 13 & 32.5 & 40 (100) & 6 & 75 & 2 & 25 & $8(100)$ \\
\hline Cotonou & 28 & 62.2 & 17 & 37.8 & $45(100)$ & 6 & 50 & 6 & 50 & $12(100)$ \\
\hline Total & 101 & 61.9 & 62 & 38.1 & $163(100)$ & 20 & 57.1 & 15 & 42.9 & $35(100)$ \\
\hline
\end{tabular}

In Natitingou, Bohicon and Cotonou, puddles were mostly encountered in urban districts $(71.1 \%, 62.1 \%$ and $60 \%$, respectively) compared to non-urban districts $(28.1 \%, 37.1 \%$ and $40 \%$, respectively) during the rainy season (Table 2 ).

\section{Larval density in the breeding sites}

The densities of Anopheles larvae in each collection period and in different districts are shown by the Table 2. In Parakou, the most productive breeding sites for Anopheles larvae were located in the urban districts including the gutters ( $18.5 \pm 23.3$ larvae/dip) followed by the tyre tracks ( $12 \pm 12.1$ larvae/dip) and the ponds ( $7.2 \pm 5.3$ larvae/dip). Similar observations were made in Natitingou with tyre tracks (6.5 \pm 2.1 larvae/dip) and ponds (5.3 \pm 8.3 larvae/dip) in urban districts like the mostly productive habitats. In Bohicon and Cotonou the highest density of larvae was respectively found in gutters (11.4 \pm 12.0 larvae/dip) and vegetable farms (6.5 \pm 4.9 larvae/dip) in the urban districts. In the non-urban districts, the highest larval density was found in the vegetable farms in the cities of 
Parakou ( $7 \pm 4.2$ larvae/dip) and Cotonou ( $6.5 \pm 4.9$ larvae/dip). In Natitingou and Bohicon, these Anopheles highest larval densities were found in rainy season's tyre tracks ( $6.5 \pm 2.1$ larvae/dip) and dry season's gutters ( $4 \pm 4.2$ larvae/dip). 
Table 2

Typology of breeding sites and their mean larval density according to the level of urbanization of the districts and different seasons

\begin{tabular}{|c|c|c|c|c|c|c|c|c|c|}
\hline \multirow[t]{2}{*}{ Seasons } & \multirow{2}{*}{$\begin{array}{l}\text { Habitat } \\
\text { types }\end{array}$} & \multicolumn{2}{|l|}{ Parakou } & \multicolumn{2}{|l|}{ Natitingou } & \multicolumn{2}{|l|}{ Bohicon } & \multicolumn{2}{|l|}{ Cotonou } \\
\hline & & $\begin{array}{l}\text { Urban } \\
\text { districts } \\
\text { n (Larval } \\
\text { density } \\
\text { /dip) }\end{array}$ & $\begin{array}{l}\text { Non-Urban } \\
\text { districts n } \\
\text { (Larval } \\
\text { density } \\
\text { /dip) }\end{array}$ & $\begin{array}{l}\text { Urban } \\
\text { districts } \\
\text { n (Larval } \\
\text { density } \\
\text { /dip) }\end{array}$ & $\begin{array}{l}\text { Non-Urban } \\
\text { districts n } \\
\text { (Larval } \\
\text { density } \\
\text { /dip) }\end{array}$ & $\begin{array}{l}\begin{array}{l}\text { Urban } \\
\text { districts }\end{array} \\
\text { n } \\
\text { (Larval } \\
\text { density } \\
\text { /dip) }\end{array}$ & $\begin{array}{l}\text { Non- } \\
\text { Urban } \\
\text { districts n } \\
\text { (Larval } \\
\text { density } \\
\text { /dip) }\end{array}$ & $\begin{array}{l}\text { Urban } \\
\text { districts } \\
\text { n (Larval } \\
\text { density } \\
\text { /dip) }\end{array}$ & $\begin{array}{l}\text { Non- } \\
\text { Urban } \\
\text { districts n } \\
\text { (Larval } \\
\text { density } \\
\text { /dip) }\end{array}$ \\
\hline \multirow[t]{15}{*}{$\begin{array}{l}\text { Rainy } \\
\text { Season }\end{array}$} & Gutters & $\begin{array}{l}2(18.5 \\
\pm 23.3)\end{array}$ & $\begin{array}{l}3(4.3 \\
\pm 4.9)\end{array}$ & 0 & $1(5)$ & $\begin{array}{l}5(11.4 \\
\pm 12.0)\end{array}$ & $1(1)$ & 0 & 0 \\
\hline & Tyres & $\begin{array}{l}5(1.5 \\
\pm 0.8)\end{array}$ & $\begin{array}{l}9(1.9 \\
\pm 1.6)\end{array}$ & $\begin{array}{l}5(2.5 \\
\pm 2.1)\end{array}$ & 0 & $\begin{array}{l}4(2.7 \\
\pm 1.7)\end{array}$ & $\begin{array}{l}2(3.5 \\
\pm 2.1)\end{array}$ & $1(2)$ & 0 \\
\hline & $\begin{array}{l}\text { Vegetable } \\
\text { farms }\end{array}$ & 0 & $2(7 \pm 4.2)$ & 0 & 0 & 0 & 0 & $\begin{array}{l}2(6.5 \\
\pm 4.9)\end{array}$ & 0 \\
\hline & Puddles & $\begin{array}{l}2(1.2 \\
\pm 0.3)\end{array}$ & $\begin{array}{l}7(4.5 \\
\pm 3.5)\end{array}$ & $\begin{array}{l}10(3.7 \\
\pm 3.1)\end{array}$ & $\begin{array}{l}4(5.2 \\
\pm 6.5)\end{array}$ & $\begin{array}{l}17(3 \\
\pm 2.1)\end{array}$ & $\begin{array}{l}10(3.4 \\
\pm 4.4)\end{array}$ & $\begin{array}{l}21(4.3 \\
\pm 4.5)\end{array}$ & $\begin{array}{l}14(2.5 \\
\pm 1.5)\end{array}$ \\
\hline & Tyre tracks & $\begin{array}{l}3(12 \\
\pm 12.1)\end{array}$ & 0 & $\begin{array}{l}2(6.5 \\
\pm 2.1)\end{array}$ & $\begin{array}{l}3(6.3 \\
\pm 3.0)\end{array}$ & 0 & 0 & 0 & $3(4 \pm 3.6)$ \\
\hline & Swamps & $1(3)$ & 0 & $1(3)$ & 0 & 0 & 0 & 0 & 0 \\
\hline & Pits & 0 & 0 & $1(3)$ & 0 & 0 & 0 & $\begin{array}{l}3(1.3 \\
\pm 0.6)\end{array}$ & 0 \\
\hline & $\begin{array}{l}\text { Animal } \\
\text { drinkers }\end{array}$ & 0 & $1(1)$ & 0 & 0 & 0 & 0 & 0 & 0 \\
\hline & $\begin{array}{l}\text { Hoof } \\
\text { imprints }\end{array}$ & $1(2)$ & 0 & 0 & $1(2)$ & 0 & 0 & 0 & 0 \\
\hline & Tin can & 0 & $1(1)$ & 0 & 0 & 0 & 0 & 0 & 0 \\
\hline & Ponds & $\begin{array}{l}6(7.2 \\
\pm 5.3)\end{array}$ & 0 & $\begin{array}{l}5(6.3 \\
\pm 8.3)\end{array}$ & 0 & $1(2)$ & 0 & 0 & 0 \\
\hline & $\begin{array}{l}\text { Abandoned } \\
\text { cans }\end{array}$ & 0 & 0 & $1(4)$ & 0 & 0 & 0 & 0 & 0 \\
\hline & Wells & 0 & 0 & $1(0.5)$ & 0 & 0 & 0 & 0 & 0 \\
\hline & $\begin{array}{l}\text { Construction } \\
\text { sites }\end{array}$ & 0 & 0 & 0 & 0 & 0 & 0 & $1(6)$ & 0 \\
\hline & Total & $\begin{array}{l}20(8.7 \\
\pm 8.9)\end{array}$ & $\begin{array}{l}23(3.4 \\
\pm 3.2)\end{array}$ & $\begin{array}{l}26(3.9 \\
\pm 4.3)\end{array}$ & $\begin{array}{l}9(5.2 \\
\pm 4.9)\end{array}$ & $\begin{array}{l}27(4.5 \\
\pm 6.0)\end{array}$ & $\begin{array}{l}13(3.3 \\
\pm 3.9)\end{array}$ & $\begin{array}{l}28(4.1 \pm \\
4.2)\end{array}$ & $\begin{array}{l}17(2.8 \\
\pm 1.9)\end{array}$ \\
\hline \multirow{8}{*}{$\begin{array}{l}\text { Dry } \\
\text { Season }\end{array}$} & & & & & & & & & \\
\hline & Gutters & $1(3)$ & $\begin{array}{l}2(4.5 \\
\pm 3.5)\end{array}$ & $1(2)$ & 0 & $\begin{array}{l}2(4.5 \\
\pm 0.7)\end{array}$ & $2(4 \pm 4.2)$ & $\begin{array}{l}2(2 \\
\pm 1.4)\end{array}$ & 0 \\
\hline & Tyres & $1(5)$ & 0 & 0 & 0 & $1(2)$ & 0 & $1(0.5)$ & 0 \\
\hline & $\begin{array}{l}\text { Vegetable } \\
\text { farms }\end{array}$ & 0 & 0 & $2(4 \pm 4.2)$ & 0 & $1(5)$ & 0 & $\begin{array}{l}2(2.5 \\
\pm 0.7)\end{array}$ & 0 \\
\hline & Puddles & 0 & $\begin{array}{l}2(2.5 \\
\pm 2.1)\end{array}$ & $\begin{array}{l}2(1.5 \\
\pm 0.70)\end{array}$ & 0 & $1(0.1)$ & 0 & 0 & $1(2)$ \\
\hline & Tyre tracks & 0 & $1(2)$ & 0 & 0 & 0 & 0 & 0 & $1(2)$ \\
\hline & $\begin{array}{l}\text { Bricks } \\
\text { Hollow }\end{array}$ & 0 & 0 & 0 & 0 & 0 & 0 & 0 & $1(0.5)$ \\
\hline & Flowerpots & 0 & 0 & 0 & 0 & 0 & 0 & $1(1)$ & 0 \\
\hline
\end{tabular}




\begin{tabular}{|c|c|c|c|c|c|c|c|c|c|}
\hline \multirow[t]{2}{*}{ Seasons } & \multirow{2}{*}{$\begin{array}{l}\text { Habitat } \\
\text { types }\end{array}$} & \multicolumn{2}{|l|}{ Parakou } & \multicolumn{2}{|c|}{ Natitingou } & \multicolumn{2}{|l|}{ Bohicon } & \multicolumn{2}{|l|}{ Cotonou } \\
\hline & & $\begin{array}{l}\text { Urban } \\
\text { districts } \\
\text { n (Larval } \\
\text { density } \\
\text { /dip) }\end{array}$ & $\begin{array}{l}\text { Non-Urban } \\
\text { districts n } \\
\text { (Larval } \\
\text { density } \\
\text { /dip) }\end{array}$ & $\begin{array}{l}\text { Urban } \\
\text { districts } \\
\text { n (Larval } \\
\text { density } \\
\text { /dip) }\end{array}$ & $\begin{array}{l}\text { Non-Urban } \\
\text { districts n } \\
\text { (Larval } \\
\text { density } \\
\text { /dip) }\end{array}$ & $\begin{array}{l}\begin{array}{l}\text { Urban } \\
\text { districts }\end{array} \\
\text { n } \\
\text { (Larval } \\
\text { density } \\
\text { /dip) }\end{array}$ & $\begin{array}{l}\text { Non- } \\
\text { Urban } \\
\text { districts n } \\
\text { (Larval } \\
\text { density } \\
\text { /dip) }\end{array}$ & $\begin{array}{l}\text { Urban } \\
\text { districts } \\
\text { n (Larval } \\
\text { density } \\
\text { /dip) }\end{array}$ & $\begin{array}{l}\text { Non- } \\
\text { Urban } \\
\text { districts n } \\
\text { (Larval } \\
\text { density } \\
\text { /dip) }\end{array}$ \\
\hline & $\begin{array}{l}\text { Under } \\
\text { bridges }\end{array}$ & 0 & $\begin{array}{l}2(3.5 \\
\pm 3.5)\end{array}$ & 0 & 0 & $1(2)$ & 0 & 0 & 0 \\
\hline & Swamps & 0 & 0 & 0 & 0 & 0 & 0 & 0 & $2(1 \pm 0)$ \\
\hline & Pits & $1(0.5)$ & 0 & 0 & 0 & 0 & 0 & 0 & $1(7)$ \\
\hline & Total & $\begin{array}{l}3(2.8 \\
\pm 2.2)\end{array}$ & $\begin{array}{l}7(3.3 \\
\pm 2.4)\end{array}$ & $\begin{array}{l}5(2.6 \\
\pm 2.1)\end{array}$ & 0 & $\begin{array}{l}6(3.0 \\
\pm 1.9)\end{array}$ & $2(4 \pm 4.2)$ & $\begin{array}{l}6(1.7 \\
\pm 1.1)\end{array}$ & $\begin{array}{l}6(2.3 \\
\pm 2.4)\end{array}$ \\
\hline$p$-value & & 0.2667 & 0.9399 & 0.5180 & - & 0.5535 & 0.8180 & 0.1789 & 0.1347 \\
\hline
\end{tabular}

During this study, the highest larval densities of Anopheles mosquitoes were observed during the rainy season in several districts, but there were no significant differences in the larval densities in the different seasons and in different districts.

\section{Nature and origin of the breeding sites}

During the collection period, the breeding sites surveyed were classified as "Temporary" and "Permanent". In the two collections periods, the majority of breeding sites were temporary in the four cities. During the rainy season, respectively $81.4 \%\left(X^{2}=11.881, d f=1, P=\right.$ $0.0006) ; 83.3 \%\left(X^{2}=10.742, d f=1, P=0.001\right) ; 97.5 \%\left(X^{2}=18.501, d f=1, P<0.0001\right)$ and $93.3 \%\left(X^{2}=18.807, d f=1, P<0.0001\right)$ habitats were temporary in Parakou, Natitingou, Bohicon and Cotonou (Table 3). During the dry season, the percentages of temporary breeding sites identified were $50 \%$ in Parakou $\left(X^{2}=0.0, d f=1, P=1.0\right), 60 \%$ in Natitingou $\left(X^{2}=0.154, d f=1, P=0.6949\right), 100 \%$ in Bohicon $\left(X^{2}=0.0, d f=1\right.$, $P=1.0)$ and $75 \%$ and in Cotonou $\left(X^{2}=2.200, d f=1, P=0.1380\right)$.

Concerning the origin of the breeding sites, they were either natural or artificial. The natural sites included puddles, ponds and swamps. However, habitats such as gutters, tyres, construction sites, tips, cans, vegetable farms, owed their origin to human activities and were classified as artificial. In Natitingou, Bohicon and Cotonou, natural sites were more abundant than artificial sites during the rainy season, with 58.1, 70 and 77.1\%, respectively. However, in Parakou, artificial sites (60.1\%) were more numerous than natural sites (39.1\%) during the same season. In the dry season, cities surveyed had high proportions of artificial sites (Table 3).

Table 3

Nature and origin of the breeding sites according to the seasons in the different cities

\begin{tabular}{|c|c|c|c|c|c|c|c|c|c|c|}
\hline \multirow[t]{3}{*}{ Cities } & \multicolumn{5}{|c|}{ Rainy season } & \multicolumn{5}{|l|}{ Dry season } \\
\hline & \multicolumn{2}{|c|}{ Nature of the site } & \multicolumn{2}{|c|}{ Origin of site } & \multirow{2}{*}{$\begin{array}{l}\text { Total } \\
\mathrm{N}(\%)\end{array}$} & \multicolumn{2}{|c|}{ Nature of site } & \multicolumn{2}{|c|}{ Origin of site } & \multirow{2}{*}{$\begin{array}{l}\text { Total } \\
\mathbf{N}(\%)\end{array}$} \\
\hline & $\begin{array}{l}\text { Temporary } \\
\text { n (\%) }\end{array}$ & $\begin{array}{l}\text { Permanent } \\
\mathrm{n}(\%)\end{array}$ & $\begin{array}{l}\text { Natural } \\
\mathrm{n}(\%)\end{array}$ & $\begin{array}{l}\text { Artificial } \\
\text { n (\%) }\end{array}$ & & $\begin{array}{l}\text { Temporary } \\
\text { n (\%) }\end{array}$ & $\begin{array}{l}\text { Permanent } \\
\mathrm{n}(\%)\end{array}$ & $\begin{array}{l}\text { Natural } \\
\text { n (\%) }\end{array}$ & $\begin{array}{l}\text { Artificial } \\
n(\%)\end{array}$ & \\
\hline Parakou & $\begin{array}{l}35 \\
(81.4)\end{array}$ & $\begin{array}{l}8 \\
(18.6)\end{array}$ & $\begin{array}{l}17 \\
(39.5)\end{array}$ & $\begin{array}{l}26 \\
(60.5)\end{array}$ & $\begin{array}{l}43 \\
(100)\end{array}$ & $\begin{array}{l}5 \\
(50)\end{array}$ & $\begin{array}{l}5 \\
(50)\end{array}$ & $\begin{array}{l}2 \\
(20)\end{array}$ & $\begin{array}{l}8 \\
(80)\end{array}$ & $\begin{array}{l}10 \\
(100)\end{array}$ \\
\hline Natitingou & $\begin{array}{l}30 \\
(83.3)\end{array}$ & $\begin{array}{l}6 \\
(16.7)\end{array}$ & $\begin{array}{l}21 \\
(58.3)\end{array}$ & $\begin{array}{l}15 \\
(41.7)\end{array}$ & $\begin{array}{l}36 \\
(100)\end{array}$ & $\begin{array}{l}3 \\
(60)\end{array}$ & $\begin{array}{l}2 \\
(40)\end{array}$ & $\begin{array}{l}2 \\
(40)\end{array}$ & $\begin{array}{l}3 \\
(60)\end{array}$ & $\begin{array}{l}5 \\
(100)\end{array}$ \\
\hline Bohicon & $\begin{array}{l}39 \\
(97.5)\end{array}$ & $\begin{array}{l}1 \\
(2.5)\end{array}$ & $\begin{array}{l}28 \\
(70)\end{array}$ & $\begin{array}{l}12 \\
(30)\end{array}$ & $\begin{array}{l}40 \\
(100)\end{array}$ & $\begin{array}{l}8 \\
(100)\end{array}$ & $\begin{array}{l}0 \\
(00)\end{array}$ & $\begin{array}{l}1 \\
(12.5)\end{array}$ & $\begin{array}{l}7 \\
(87.5)\end{array}$ & $\begin{array}{l}8 \\
(100)\end{array}$ \\
\hline Cotonou & $\begin{array}{l}42 \\
(93.3)\end{array}$ & $\begin{array}{l}3 \\
(6.7)\end{array}$ & $\begin{array}{l}35 \\
(77.8)\end{array}$ & $\begin{array}{l}10 \\
(22.2)\end{array}$ & $\begin{array}{l}45 \\
(100)\end{array}$ & $\begin{array}{l}9 \\
(75)\end{array}$ & $\begin{array}{l}3 \\
(25)\end{array}$ & $\begin{array}{l}3 \\
(25)\end{array}$ & $\begin{array}{l}9 \\
(75)\end{array}$ & $\begin{array}{l}12 \\
(100)\end{array}$ \\
\hline
\end{tabular}




\section{Effect of physicochemical parameters on the productivity of breeding sites}

The tables 4 and 5 show the mean physicochemical characteristics of different breeding sites types in rainy and dry seasons. During the rainy season, the highest temperature $\left(39.9^{\circ} \mathrm{C}\right)$ and the lowest one $\left(24.1^{\circ} \mathrm{C}\right)$ were recorded in pound and tyre in Parakou. The pH ranged from 2.88 to 10.44 . The lowest value of $\mathrm{pH}$ was obtained in vegetable farm and highest value in puddle during the reany saison in the city of Parakou. The dissolved oxygen was high $(8.23 \mathrm{mg} / \mathrm{L})$ in swamp in Natitingou and low in gutter in Bohicon. The highest values of salinity $(0.8 \mathrm{~g} / \mathrm{L})$ and conductivity $(1435 \mu \mathrm{S} / \mathrm{cm})$ were both measured during the rainy season in tyre tracks at Parakou. The lowest salinity $(0.1 \mathrm{~g} / \mathrm{L})$ was recorded in large habitat sites while the lowest conductivity $(43.2 \mu \mathrm{S} / \mathrm{cm})$ was recorded in tyres during the rainy season in Bohicon. About the turbidity of larvae habitats, the highest value was 970 NTU in gutter while the lowest value was 1.35 NTU in tyres encountered in Bohicon during the rainy season.

Table 4

Physicochemical parameters of breeding sites in the rainy season in the study sites

\begin{tabular}{|c|c|c|c|c|c|c|c|c|c|c|}
\hline \multirow{2}{*}{$\begin{array}{l}\text { Breeding } \\
\text { sites }\end{array}$} & \multirow[t]{2}{*}{$\mathbf{n}$} & \multicolumn{7}{|c|}{ Physicochemical parameters } & \multirow{2}{*}{$\begin{array}{l}\text { Depth } \\
\text { (m) }\end{array}$} & \multirow{2}{*}{$\begin{array}{l}\text { area } \\
\left(\mathrm{m}^{2}\right)\end{array}$} \\
\hline & & $\begin{array}{l}\text { Temperature } \\
\left({ }^{\circ} \mathrm{C}\right)\end{array}$ & $\mathrm{Ph}$ & $\begin{array}{l}\text { Dissolved } \\
\text { oxygen } \\
\text { (mg/L) }\end{array}$ & $\begin{array}{l}\text { Conductivity } \\
(\mu \mathrm{S} / \mathrm{cm})\end{array}$ & $\begin{array}{l}\text { Salinity } \\
(g / L)\end{array}$ & $\begin{array}{l}\text { TDS } \\
(\mathrm{ppm})\end{array}$ & $\begin{array}{l}\text { Turbidity } \\
\text { (NTU) }\end{array}$ & & \\
\hline Gutters & 12 & $30.86 \pm 3.14$ & $\begin{array}{l}7.13 \\
\pm 0.10\end{array}$ & $2.19 \pm 1.12$ & $\begin{array}{l}536.11 \\
\pm 329.18\end{array}$ & $\begin{array}{l}0.13 \\
\pm 1.16\end{array}$ & $\begin{array}{l}388.16 \\
\pm 235.13\end{array}$ & $\begin{array}{l}240.16 \\
\pm 338.18\end{array}$ & $\begin{array}{l}0.16 \\
\pm 0.07\end{array}$ & $\begin{array}{l}1.13 \\
\pm 0.10\end{array}$ \\
\hline Tyres & 14 & $27.15 \pm 2.18$ & $\begin{array}{l}7.15 \\
\pm 0.12\end{array}$ & $2.10 \pm 1.15$ & $\begin{array}{l}401.12 \\
\pm 296.12\end{array}$ & $\begin{array}{l}0.11 \\
\pm 0.18\end{array}$ & $\begin{array}{l}247.13 \\
\pm 190.18\end{array}$ & $\begin{array}{l}33.16 \\
\pm 34.16\end{array}$ & $\begin{array}{l}0.07 \\
\pm 0.03\end{array}$ & $\begin{array}{l}0.12 \\
\pm 0.109\end{array}$ \\
\hline $\begin{array}{l}\text { Vegetable } \\
\text { farms }\end{array}$ & 4 & $31.12 \pm 5.12$ & $\begin{array}{l}6.15 \\
\pm 2.10\end{array}$ & $2.19 \pm 0.15$ & $\begin{array}{l}525.1 \\
\pm 420.11\end{array}$ & $\begin{array}{l}0.15 \\
\pm 0.17\end{array}$ & $\begin{array}{l}364.15 \\
\pm 299.11\end{array}$ & $\begin{array}{l}20.17 \\
\pm 26.19\end{array}$ & $\begin{array}{l}0.06 \\
\pm 0.03\end{array}$ & $\begin{array}{l}0.15 \\
\pm 1.14\end{array}$ \\
\hline Puddles & 78 & $31.15 \pm 3.10$ & $\begin{array}{l}8.19 \\
\pm 0.18\end{array}$ & $2.10 \pm 1.13$ & $\begin{array}{l}302.14 \\
\pm 218.14\end{array}$ & $\begin{array}{l}0.13 \\
\pm 0.19\end{array}$ & $\begin{array}{l}184.12 \\
\pm 144.16\end{array}$ & $\begin{array}{l}179.12 \\
\pm 220.14\end{array}$ & $\begin{array}{l}0.12 \\
\pm 0.13\end{array}$ & $\begin{array}{l}3.18 \\
\pm 1.14\end{array}$ \\
\hline $\begin{array}{l}\text { Animal } \\
\text { drinker }\end{array}$ & 1 & 25.1 & 9.13 & 2.15 & 540 & 0.1 & 447.1 & 109 & 0.2 & 0.19 \\
\hline $\begin{array}{l}\text { Hoof } \\
\text { imprints }\end{array}$ & 2 & $32.15 \pm 8.19$ & $\begin{array}{l}7.12 \\
\pm 0.17\end{array}$ & $2.19 \pm 2.17$ & $\begin{array}{l}475 \\
\pm 200.11\end{array}$ & $\begin{array}{l}0.15 \\
\pm 0.17\end{array}$ & $\begin{array}{l}295.1 \\
\pm 87.11\end{array}$ & $\begin{array}{l}562.1 \\
\pm 118.18\end{array}$ & 0.1 & $\begin{array}{l}0.14 \\
\pm 0.19\end{array}$ \\
\hline Tin cans & 1 & 28.1 & 9.14 & 0.1 & 922 & 0.1 & 507 & 38.12 & 0.05 & 0.13 \\
\hline Ponds & 12 & $30.15 \pm 3.14$ & $\begin{array}{l}7.16 \\
\pm 0.15\end{array}$ & $3.19 \pm 1.12$ & $\begin{array}{l}350.16 \\
\pm 205.12\end{array}$ & $\begin{array}{l}0.16 \\
\pm 0.11\end{array}$ & $\begin{array}{l}224.14 \\
\pm 142.17\end{array}$ & $\begin{array}{l}113.17 \\
\pm 163.18\end{array}$ & $\begin{array}{l}0.22 \\
\pm 0.20\end{array}$ & $\begin{array}{l}2.17 \\
\pm 0.10\end{array}$ \\
\hline Tyre tracks & 11 & $30.1 \pm 3.12$ & $\begin{array}{l}7.17 \\
\pm 0.15\end{array}$ & $2.15 \pm 1.17$ & $\begin{array}{l}602.1 \\
\pm 520.17\end{array}$ & $\begin{array}{l}0.17 \\
\pm 0.19\end{array}$ & $\begin{array}{l}416.13 \\
\pm 392.17\end{array}$ & $\begin{array}{l}255.18 \\
\pm 246.19\end{array}$ & $\begin{array}{l}0.08 \\
\pm 0.03\end{array}$ & $\begin{array}{l}0.16 \\
\pm 0.16\end{array}$ \\
\hline Swamps & 2 & $30.1 \pm 5.13$ & $\begin{array}{l}7.17 \\
\pm 0.17\end{array}$ & $7.19 \pm 0.19$ & $\begin{array}{l}373.1 \\
\pm 111.19\end{array}$ & $\begin{array}{l}0.15 \\
\pm 0.17\end{array}$ & $\begin{array}{l}232.15 \\
\pm 79.17\end{array}$ & $\begin{array}{l}39.13 \\
\pm 22.19\end{array}$ & $\begin{array}{l}0.15 \\
\pm 0\end{array}$ & $\begin{array}{l}1.12 \\
\pm 0.19\end{array}$ \\
\hline Pits & 3 & $30.1 \pm 0.18$ & $\begin{array}{l}7.16 \\
\pm 0.16\end{array}$ & $3.15 \pm 1.18$ & $\begin{array}{l}603.16 \\
\pm 329.19\end{array}$ & $\begin{array}{l}0.13 \\
\pm 0.15\end{array}$ & $\begin{array}{l}377.15 \\
\pm 217.18\end{array}$ & $\begin{array}{l}9.17 \\
\pm 1.19\end{array}$ & $\begin{array}{l}0.55 \\
\pm 0.42\end{array}$ & $\begin{array}{l}2.18 \\
\pm 1.14\end{array}$ \\
\hline $\begin{array}{l}\text { Construction } \\
\text { sites }\end{array}$ & 1 & 27.1 & 8.15 & 3.12 & 220.1 & 0.1 & 139.1 & 17.18 & 0.03 & 4.11 \\
\hline
\end{tabular}


Table 5

Physicochemical parameters of breeding sites in dry season in the study sites

\begin{tabular}{|c|c|c|c|c|c|c|c|c|c|c|}
\hline \multirow{2}{*}{$\begin{array}{l}\text { Breeding } \\
\text { sites }\end{array}$} & \multirow[t]{2}{*}{$\mathbf{N}$} & \multicolumn{7}{|c|}{ Physicochemical parameters } & \multirow{2}{*}{$\begin{array}{l}\text { Depth } \\
\text { (m) }\end{array}$} & \multirow{2}{*}{$\begin{array}{l}\text { area } \\
\left(\mathrm{m}^{2}\right)\end{array}$} \\
\hline & & $\begin{array}{l}\text { Température } \\
\left({ }^{\circ} \mathrm{C}\right)\end{array}$ & $\mathrm{pH}$ & $\begin{array}{l}\text { Dissolved } \\
\text { oxygen } \\
\text { (mg/L) }\end{array}$ & $\begin{array}{l}\text { Conductivity } \\
(\mu \mathrm{S} / \mathrm{cm})\end{array}$ & $\begin{array}{l}\text { Salinity } \\
(g / L)\end{array}$ & $\begin{array}{l}\text { TDS } \\
\text { (ppm) }\end{array}$ & $\begin{array}{l}\text { Turbidity } \\
\text { (NTU) }\end{array}$ & & \\
\hline Gutters & 10 & $30.6 \pm 2.12$ & $\begin{array}{l}8.10 \\
\pm 0.11\end{array}$ & $2.11 \pm 1.1$ & $\begin{array}{l}583.1 \\
\pm 179.1\end{array}$ & $\begin{array}{l}0.14 \\
\pm 0.17\end{array}$ & $\begin{array}{l}203.1 \\
\pm 193.1\end{array}$ & $\begin{array}{l}24.13 \\
\pm 4.13\end{array}$ & $\begin{array}{l}0.11 \\
\pm 0.16\end{array}$ & $\begin{array}{l}1.16 \\
\pm 1.16\end{array}$ \\
\hline Tyres & 3 & $31.1 \pm 2.14$ & $\begin{array}{l}8.15 \\
\pm 0.12\end{array}$ & $0.19 \pm 1.1$ & $\begin{array}{l}285.18 \\
\pm 333.1\end{array}$ & $\begin{array}{l}0.19 \\
\pm 0.11\end{array}$ & $\begin{array}{l}182.1 \\
\pm 137.1\end{array}$ & $\begin{array}{l}17.18 \\
\pm 13.10\end{array}$ & $\begin{array}{l}0.06 \\
\pm 0.001\end{array}$ & $\begin{array}{l}0.12 \\
\pm 0.107\end{array}$ \\
\hline $\begin{array}{l}\text { Vegetable } \\
\text { farms }\end{array}$ & 5 & $29.1 \pm 1.14$ & $\begin{array}{l}8.18 \\
\pm 0.19\end{array}$ & $\begin{array}{l}2.15 \\
\pm 2.12\end{array}$ & $\begin{array}{l}621.1 \\
\pm 290.1\end{array}$ & $\begin{array}{l}0.12 \\
\pm 0.18\end{array}$ & $\begin{array}{l}307.1 \\
\pm 261.1\end{array}$ & $\begin{array}{l}10.10 \\
\pm 6.18\end{array}$ & $\begin{array}{l}0.16 \\
\pm 0.16\end{array}$ & $\begin{array}{l}2.18 \\
\pm 1.18\end{array}$ \\
\hline Puddles & 6 & $31.1 \pm 10.1$ & $\begin{array}{l}7.11 \\
\pm 0.18\end{array}$ & $\begin{array}{l}1.10 \\
\pm 1.18\end{array}$ & $\begin{array}{l}267.1 \\
\pm 214.1\end{array}$ & $\begin{array}{l}0.12 \\
\pm 0.15\end{array}$ & $\begin{array}{l}187.1 \\
\pm 98.1\end{array}$ & $\begin{array}{l}106.10 \\
\pm 154.17\end{array}$ & $\begin{array}{l}0.15 \\
\pm 0.07\end{array}$ & $\begin{array}{l}2.15 \\
\pm 0.19\end{array}$ \\
\hline Tyre tracks & 2 & $35.1 \pm 1.13$ & $\begin{array}{l}7.15 \\
\pm 0.14\end{array}$ & $\begin{array}{l}1.19 \\
\pm 1.15\end{array}$ & $\begin{array}{l}791.1 \\
\pm 415.14\end{array}$ & $0.1 \pm 0.17$ & $\begin{array}{l}316.1 \\
\pm 211.1\end{array}$ & $\begin{array}{l}75.15 \\
\pm 18.11\end{array}$ & $0.08 \pm 0.02$ & $\begin{array}{l}0.1 \\
\pm 0.12\end{array}$ \\
\hline $\begin{array}{l}\text { Bricks } \\
\text { Hollow }\end{array}$ & 1 & 31.1 & 6.19 & 2.14 & 333 & 0.1 & 233.1 & 36.16 & 0.10 & 0.15 \\
\hline Flowerpots & 1 & 29.1 & 7.13 & 1.1 & 137.1 & 0.1 & 100.1 & 6.12 & 0.10 & 0.17 \\
\hline $\begin{array}{l}\text { Under } \\
\text { bridges }\end{array}$ & 3 & $30.16 \pm 1.10$ & $\begin{array}{l}7.11 \\
\pm 0.18\end{array}$ & $\begin{array}{l}1.15 \\
\pm 1.11\end{array}$ & $\begin{array}{l}523.1 \\
\pm 197.1\end{array}$ & $\begin{array}{l}0.12 \\
\pm 0.11\end{array}$ & $\begin{array}{l}404.1 \\
\pm 113.1\end{array}$ & $\begin{array}{l}35.17 \\
\pm 20.19\end{array}$ & $\begin{array}{l}0.50 \\
\pm 0.45\end{array}$ & $\begin{array}{l}7.13 \\
\pm 0.10\end{array}$ \\
\hline Swamps & 2 & $32.1 \pm 0.11$ & $\begin{array}{l}7.11 \\
\pm 0.17\end{array}$ & $\begin{array}{l}1.12 \\
\pm 0.19\end{array}$ & $\begin{array}{l}453.1 \\
\pm 250.1\end{array}$ & $0.1 \pm 0$ & 185.1 & $\begin{array}{l}195.16 \\
\pm 17.17\end{array}$ & $\begin{array}{l}0.25 \\
\pm 0.07\end{array}$ & $\begin{array}{l}12.16 \\
\pm 1.16\end{array}$ \\
\hline Pits & 2 & $34.1 \pm 3.11$ & $\begin{array}{l}7.18 \\
\pm 1.11\end{array}$ & $\begin{array}{l}1.14 \\
\pm 0.11\end{array}$ & $\begin{array}{l}381.1 \\
\pm 127.1\end{array}$ & $0.15 \pm 0.17$ & $\begin{array}{l}243.1 \\
\pm 114.1\end{array}$ & $\begin{array}{l}33.16 \\
\pm 26.11\end{array}$ & $\begin{array}{l}0.25 \\
\pm 0.07\end{array}$ & $\begin{array}{l}5.13 \\
\pm 7.13\end{array}$ \\
\hline
\end{tabular}

Principal Component Analysis (PCA) was used to determine the relationships between the investigated physicochemical variables and Anopheles larval productivity. The projections revealed that the larval productivity was linked to variations of some physicochemical parameters (Figure 4). Indeed, in the city of Parakou, the larval density was related to the 2nd component-axis and showed positive correlations with dissolved oxygen, $\mathrm{pH}$ and temperature. These positive correlations were statistically significant with dissolved oxygen $(r=0.26, P=0.038)$ and temperature $(r=0.23 P=0.047)$. In the city of Natitingou, there was no significant correlation between physicochemical characteristics and the abundance of Anopheles larvae. In Bohicon, dissolved oxygen was related to larval density in the first component-axis but had no significant effects $(r=0.16, P=0.862)$. In the city of Cotonou, temperature $(r=0.23, P=0.048)$ and salinity $(r=0.44, P=0.002)$ were positively correlated with the density of Anopheles larvae.

\section{Effect of microbiological parameters on the productivity of breeding sites}

To determine whether bacterial composition could influence larval density, microbiological analysis of water samples from some habitats encountered in the study sites was performed. It allowed isolation, identification and count of Faecal Coliforms (FC), Total Coliforms (TC) and Escherichia coli present in the samples. Results showed high load of these bacteria in several water samples from larval habitats. Faecal Coliforms, Total Coliforms and Escherichia coli average loads were $2.1810^{5} \mathrm{UFC} / 100 \mathrm{~mL}, 1.8010^{4} \mathrm{UFC} / 100 \mathrm{~mL}$, $2.2010^{4} \mathrm{UFC} / 100 \mathrm{~mL}$ in Parakou, $6.6010^{5} \mathrm{UFC} / 100 \mathrm{~mL}, 3.3010^{4} \mathrm{UFC} / 100 \mathrm{~mL}, 9.4010^{4} \mathrm{UFC} / 100 \mathrm{~mL}$ in Natitingou, $3.9010^{5} \mathrm{UFC} / 100$ $\mathrm{mL}, 4.4010^{4} \mathrm{UFC} / 100 \mathrm{~mL}, 5.9510^{4} \mathrm{UFC} / 100 \mathrm{~mL}$ in Bohicon and $6.7610^{5} \mathrm{UFC} / 100 \mathrm{~mL}, 9.9010^{4} \mathrm{UFC} / 100 \mathrm{~mL}, 1.2610^{5} \mathrm{UFC} / 100 \mathrm{~mL}$ in Cotonou, respectively.

The figure 5 shows the cross-analysis results between bacteriological load and larval densities of the water samples in the different cities. A negative correlation was observed between the larval density and the concentration of Coliforms $(r=-0.72, P=0.0024)$. So, a negative but non-significant correlation existed between larval density and Total Coliform load $(r=-0.22, P=0.215)$. However, the results showed a weakly correlated between $E$. coli load and the larval density of the breeding sites $(r=0.06, P=0.419)$. 
The present survey was carried out to study the typology of breeding sites as well as the physicochemical and microbiological variables favourable to Anopheles larvae development in four cities in Benin. The breeding sites of mosquitoes in different areas were mainly formed as a result of human activities and urbanization related factors. These are excellent breeding habitats for Anopheles larvae. During this study, seventeen different types of habitats have been identified, ranging from puddles to abandoned tyres, including construction sites and vegetable farms. This diversity of habitats for Anopheles larvae has already been observed by Djegbe et al. in 2019 [29] in southern Benin and by many other authors in Africa [30-33]. Puddles were the main breeding sites recorded in the different study sites. These puddles were generally found in alleys or on the outskirts of the streets, which essentially denotes a lack of rainwater sanitation project from the public authorities and a poorly controlled urbanization process in these cities [34, 35]. In addition, the neglect of the local population and the economic activity practices could be the source of many types of habitats [32, 36]. Thus, the abundance of tyres serving as larval habitats for Anopheles in Parakou could be due to the increase in the transport of large trucks which serve other cities in the north and the neighbouring countries, transiting through Parakou. This leads to abandoned tyres in the city's many garages and vulcanization centres.

In addition, the classification of breeding sites as "Natural" or "Artificial" sites enables to highlight the role of humans through routine activities in maintaining the transmission of malaria in the dry season. Indeed, though the number of larval habitats is considerably reduced during this season, those found are mainly anthropogenic. Therefore, it is important to draw the attention of the population on their role in reducing the transmission of malaria through sanitation of their environment and elimination of these shelters [33].

In this study, the larval habitats were also classified as "Permanent" or "Temporary". This clustering of habitats helps understand their stability and the extent to which they contribute to the abundance of Anopheles populations during seasonal changes in the study areas [37]. A high number of temporary habitat types was observed during the two collection periods in the four cities. As the temporary breeding sites depend mainly on rain and dry out quite quickly due to the drop in precipitation, their high proportions observed in the dry season could be due to anthropogenic activities. These results were in contrast to those obtained by Kudom in 2015 who observed a high proportion of permanent habitats in the cities of Accra and Sekondi-Takoradi during the rainy and dry seasons [36]. On the other hand, these results were in line with those of Koumba et al., (2018) who obtained a high proportion of temporary sites both during the rainy and dry seasons in the agricultural areas of the Mouila zone in Gabon [30].

In three of the four cities surveyed, including Natitingou, Bohicon and Cotonou, the majority of Anopheles breeding sites were found in urban districts. This could be explained by the fact that in urban districts, breeding sites are accessible and easily spotted, unlike districts with low urban potential [29]. In addition, urban districts offer a multiplicity of typical breeding sites (temporary, sunny and clear) favourable to Anopheles, contrary to non-urban districts characterized by unsanitary conditions, and where potential breeding sites found are dominated by pollution less favourable to Anopheles development [38, 39]. These results are interesting because they justify the existence of a fairly high urban transmission of malaria, which a few years ago was still controversial [40].

In Bohicon and Cotonou, tyre tracks were the most productive habitat types. They were the second most productive in Parakou city. This can be explained by the fact that tyre tracks constitute the ideal sites for oviposition of Anopheles females due to their small surface area ( $0.76 \pm 0.36 \mathrm{~m} 2$ and $0.7 \pm 0.42 \mathrm{~m} 2$ in the rainy and dry seasons, respectively), their adequate exposure to sunlight and the absence of plant debris on their surface [41,42]. Other than tyre tracks, puddles, vegetable farms and gutters were among the habitat types with the highest larval densities in the four cities. Mattah et al., (2017) found similar results with puddles (13.7 larvae/dip), gutters (12.9/dip) and urban farm sites (11.6/dip) as most productive larval habitats of Anopheles in southern Ghana [34].

It is also important to note that the larval abundance of the different types of habitats is mainly influenced by the seasons and the physicochemical parameters. The results showed a seasonal variation in larval densities with higher density in the rainy season compared to the dry season in all types of breeding sites. It is therefore appropriate that the various control strategies to reduce malaria transmission should be intensified during the dry seasons [43].

Moreover, the physicochemical characterization of the habitats made it possible to positively correlate the density of Anopheles larvae with certain parameters including temperature, oxygen level and $\mathrm{pH}$. The positive tropism of Anopheles larvae with temperature has already been shown by several authors [44, 45,46]. According to Muturi et al. [46], low water temperatures cause a decline in the growth of microorganisms on which mosquito larvae feed. In addition, higher temperatures can be detrimental to the presence of many aquatic arthropods, including predators, increasing the chances of survival of Anopheles larvae. [13]. Temperature could therefore be a factor accelerating the development of larval stages. 
Regarding the positive correlation of Anopheles larval density with the dissolved oxygen concentration, similar results were obtained by Mahamane et al. [4] and Mbidda et al. [47]. Anopheles larvae not having a breathing siphon, dissolved oxygen in the water could theoretically be an important factor for their survival. Other authors have shown that anopheline larvae prefer fresh, well-oxygenated water with low mineral content [19]. It should nevertheless be notified an inclination of the Anopheles larvae to salinity in the city of Cotonou. This positive correlation of the density of Anopheles larvae with the salinity observed in Cotonou suggests a tolerance of this species to unusual habitats. Cotonou being a coastal city, its proximity to the sea is believed to be the source of sometimes high salt levels in certain water points in the city, to which Anopheles gambiae would be suitable. In addition, anthropogenic factors, which are even more accentuated in Cotonou due to the high demography and the development of industrial activities which could affect the quality of the water in the larval habitats. These observations are in agreement with the findings of many authors [36, 48], who found that the Anopheles larvae present in brackish roosts in Cape Coast (Ghana) were mainly of the subspecies Anopheles coluzzii. Subsequent work, taking into account molecular identification will be carried out in order to analyse the distribution of the subspecies of the Anopheles gambiae complex within the different types of breeding sites identified.

Microbiological analysis has identified the presence of faecal and total coliforms at various concentrations in the water samples from the breeding sites. The presence of these bacteria in the water indicates faecal contamination of the breeding sites which may be of human or animal origin. Furthermore, the negative correlation was observed between Anopheles larvae and the concentrations of faecal coliforms, suggesting that these coliforms inhibit the growth of Anopheles larvae [19]. While bacteria are known to be a source of nutrients in the growth of mosquito larvae, they may also be indicative of environmental conditions favourable to the presence or absence of larvae in breeding sites [49]. For example, Dada et al. [50] showed that the abundance of Escherichia coli in breeding sites was significantly negatively correlated with the density of Aedes aegypti larvae. Experimental work in the laboratory will be carried out to better understand mechanisms associated with this antilarval activity of faecal coliforms. The results on the potential role of these bacteria in hindering the survival of Anopheles larvae could be useful in different aspects of larval control.

\section{Conclusions}

This study showed that many factors such as climatic conditions and anthropogenic activities are the causes of larval proliferation and maintain the presence of artificial breeding sites throughout the year. The abundance of Anopheles larvae has been positively associated with physicochemical determinants including high temperatures and dissolved oxygen levels, and negatively with faecal coliform concentrations that are likely to inhibit the growth of anopheles larvae. Additional work should be carried out to further elucidate the antilarval activity of faecal coliforms and their possible integration as a biological larvicide in vector control strategies.

\section{Abbreviations}

IRS: Indoor Residual Spraying; LLINs: Long-Lasting Insecticidal Nets; TDS: Total Dissolved Solids; PCA: Principal Component Analysis

\section{Declarations}

\section{Ethics approval and consent to participate}

Not applicable.

\section{Consent for publication}

Not applicable

\section{Availability of data and materials}

The data sets analysed during this study are available from the corresponding author (ID) on reasonable request.

\section{Competing interests}

The authors declare that they have no competing interests.

\section{Funding}




\section{Contributions from the authors}

ID designed the study. ON, DHD, ID carried out the collection of mosquito larvae and the recording of physicochemical parameters in the breeding sites. ON, DHD conducted the microbiological analyses. ON, DHD, GT analysed all the data. LD was of assistance in the acquisition of the work materials. SC, FC, MA provided advice during the design of the study and helped to get the job done. DHD wrote the manuscript which has been critically reviewed by GT, KMAD and ID. All authors have read and approved the final version of the manuscript.

\section{Acknowledgements}

Authors acknowledge Pulchérie AGOSSANOU and Abel IDIOSSOU for technical assistance during mosquito rearing and bioassay performing.

\section{References}

1. Mathanga DP, Tembo AK, Mzilahowa T, Bauleni A, Mtimaukenena K, Taylor TE et al. Patterns and determinants of malaria risk in urban and peri-urban areas of Blantyre, Malawi. Malar J. 2016; 15:590 DOI 10.1186/s12936-016-1623-9

2. Kudom AA, Mensah BA, Agyeman TK: Characterization of mosquito larval habitats and assessment of insecticide-resistance status of Anopheles gambiae senso lato in urban areas in southwestern Ghana. J Vector Ecol. 2012;37 (1):17-82.

3. Fonds des Nations Unies pour l'enfance, Afrique Génération 2030. Favoriser les investissements dans l'enfance pour bénéficier du dividende démographique. Résumé Exécutif. 2021. https://data.unicef.org/resources/generation-2030-africa-2-0

4. Mahamane Iro S, Seydou YA, Doumma A. Mesures des indicateurs de prolifération des larves de moustiques au niveau des mares permanentes et semi permanentes de Saga, Niger. Int J Biol Chem Sci. 2020;14(4).1188-1202.

5. WHO. World Malaria Report 2018. Geneva: World Health Organisation; 2018.

http://www.who.int/malaria/publications/world_malaria_report_2018/report/en/.

6. Akogbeto M, Padonou GG, Bankole HS, Gazard DK, Gbedjissi GL. Dramatic decrease in Malaria transmission after large-scale indoor residual spraying with bendiocarb in Benin, an area of high resistance of Anopheles gambiae to Pyrethroids. Am J Trop Med Hyg. 2011;85: 586-93.

7. Ossè R, Aikpon R, Padonou GG, Oussou O, Yadouléton A, Akogbéto M. Evaluation of the efficacy of bendiocarb in indoor residual spraying against pyrethroid resistant malaria vectors in Benin: results of the third campaign. Parasit Vectors. 2012;8.1:163.

8. Rowland M, Boko P, Odjo A, Asidi A, Akogbeto M, N'Guessan R: A new long-lasting indoor residual formulation of the organophosphate insecticide pirimiphos-methyl for prolonged control of pyrethroid-resistant mosquitoes: an experimental hut trial in Benin. PLoS One. 2013;8:e69516.

9. Akoton R, Tchigossou GM, Djegbe I, Yessoufou A, Atoyebi SM, Tossou E, et al. Experimental huts trial of the efficacy of pyrethroids/piperonyl butoxide (PBO) nets treatments for controlling multi-resistant populations of Anopheles funestus s.s. in Kpomè, Southern Benin. Wellcome Open Res. 2018;3:71 DOI: 10.12688/wellcomeopenres.14589.1.

10. Djouaka R, Riveron JM, Yessoufou A, Tchigossou G, Akoton R, Irving H, et al. Multiple insecticide resistance in an infected population of the malaria vector Anopheles funestus in Benin. Parasit Vectors. 2016;9:453.

11. Djegbe I, Missihoun A, Djouaka R, Akogbeto M : Surveillance Entomologique: Dynamique de la population et de la résistance aux insecticides chez Anopheles gambiae s.I en milieu de riziculture irriguée au Sud Bénin. J Appl Biosci. 2017;111: 10934-10943.

12. WHO. World Malaria report 2019. Geneva: World Health Organization; 2019. http://www.who.int/malaria/fr

13. Soleimani-Ahmadi $\mathrm{M}$, Vatandoost $\mathrm{H}$, Zare M: Characterization of larval habitats for anopheline mosquitoes in a malarious area under elimination program in the southeast of Iran. Asian Pac J Trop Biomed. 2014;4(Suppl1):S73-S80.

doi:10.12980/APJTB.4.2014C899.

14. Floore TG: Mosquito Larval Control Practices: Past and Present. J Am Mosq Control Assoc. 2006;22(3):527-533.

15. WHO. World Malaria report 2016. Geneva, Switzerland, World Health Organization. 2016a.

http://www.who.int/malaria/publications/world-malaria-report-2016. 
16. Nikookar SH, Fazeli-Dinan M, Azari-Hamidian S, Mousavinasab SN, Aarabi M, Ziapour SP, et al. Correlation between mosquito larval density and their habitat physicochemical characteristics in Mazandaran Province, northern Iran. PLoS Negl Trop Dis. 2017;11(8): e0005835. https://doi.org/10.1371/journal.pntd.0005835

17. Wang H, Wang Y, Cheng P, Wang H, Wang H, Liu H, et al. The Larval Density of Mosquitos (Diptera: Culicidae) in Jiaxiang County, Shandong Province, China: Influence of Bacterial Diversity, Richness, and Physicochemical Factors. Front. Ecol. Evol. 2021;9:616769.doi: 10.3389/fevo.2021.616769

18. Omolade 00, Adetutu SA: Oviposition and Breeding Water Sites Preferences of Mosquitoes within Ojo area, Lagos State, Nigeria. Biomed J Sci\&Tech Res. 2018;7(5)-BJSTR MS.ID.001565. DOI: 10.26717/ BJSTR.2018.07.001565.

19. El Ouali Lalami A, El hilali O, Benlamlih M, Merzouki M, Raiss N, Ibensouda Koraichi S, et al. Etude entomologique, physicochimique et bactériologique des gîtes larvaires de localités à risque potentiel pour le paludisme dans la ville de Fès. Bull Inst Sci Rabat. 2010, n³2 (2).119-127.

20. INSAE. Effectifs de la population des villages et quartiers de ville du Benin (RGPH-4, 2013). Porto-Novo, Benin: Institut National de la Statistique et de l'analyse Economique; 2016.

21. Kora O. Monographie de la commune de Parakou. Programme d'appui au démarrage des communes, Afrique Conseil. 2006 ; 44 p.

22. Biaou CF. Monographie de la commune de Natitingou. Programme d'appui au démarrage des communes, Afrique Conseil. 2006;85 p.

23. Houngnihin R. Monographie de la commune de Bohicon. Programme d'appui au démarrage des communes, Afrique Conseil. 2006;25 p.

24. Akomagni L. Monographie de la commune de Cotonou. Programme d'appui au démarrage des communes, Afrique Conseil. 2006;47 p.

25. Silver JB, Service MW. Mosquito Ecology: Field sampling methods. Springer Eds. 2007;1494 pp

26. Talipouo A, Ntonga-Akono P, Tagne D, Mbida-Mbida A, Etang J, Tchoffo-Fobasso R, et al. Comparative study of Culicidae biodiversity of Manoka island and Youpwe mainland area, Littoral, Cameroon. Int J of Biosci. 2017;10(4):9-18. DOI : http://dx.doi.org/10.12692/ijb/10.4.9-18

27. Gillies MT, De Meillon B: The Anophelinae of Africa south of the Sahara (Ethiopian Zoogeographical Region). Publ S Afr Inst Med Res. 1968; 54: -343.

28. Nonfodji OM, Fatombi JK, Ahoyo TA, Boya B, Baba-Moussa LS, Aminou T. Effect of $\mathrm{KMnO}_{4}$ amounts on antibacterial property of activated carbon for efficient treatment of northern Benin hospital wastewater in a fixed bed column system. Int $\mathrm{J}$ Hyg Environ Health. 2020; 229: 113581.

29. Djègbè I, Toponon F, Gbankoto A, Tchigossou G, Djossou-Hessou D, Dossou C, et al. Typologie des gîtes larvaires et résistance des vecteurs du paludisme à la deltaméthrine dans les milieux urbain et rural du département de l'atlantique au sud du Benin : données préliminaires. Euro Sci J. November 2019; edition Vol.15, No.33 ISSN: 1857 - 7881 (Print) e - ISSN $1857-7431$. Doi:10.19044/esj.2019.v15n33p171 URL:http://dx.doi.org/10.19044/esj.2019.v15n33p171

30. Koumba AA, Zinga Koumba CR, Mintsa Nguema R, Djogbenou LS, Obame Ondo P, Ketoh GK, et al. Distribution spatiale et saisonnière des gîtes larvaires des moustiques dans les espaces agricoles de la zone de Mouila, Gabon. Int. J Biol Chem Sci. 2018;10(3):1037-1047. DOI: https://dx.doi.org/10.4314/ijbcs.v12i4.19

31. Ntonga-Akono P, Mbida Mbida A, Awono AP, Youmbi EL, Abdel Kayoum Y, Kekeunou S. Habitats larvaires et sensibilité des vecteurs du paludisme aux insecticides dans des localités (semi-urbaine et rurale) de la région du littoral Camerounais : données préliminaires. Rev Ecol (Terre et Vie). 2018;Vol. 73 (2), 2018 : 132-141.

32. Tia E, Gbalégba NGC, M’Bra KR, Kaba A, Boby OAM, Koné M, et al. Étude du niveau de production larvaire d’Anopheles gambiae s.I. (Diptera: Culicidae) dans différents types de gîtes à Oussou-yaokro au Centre-Ouest et à Korhogo, au Nord (Côte d’Ivoire). J Appl Biosci. 2016;105:10170 -10182. ISSN 1997-5902.

33. Hinne I, Attah SA, Mensah BA, Forson AO, Afrane YA. Larval habitat diversity and Anopheles mosquito species distribution in different ecological zones in Ghana. Parasit Vectors. 2021;14:193. https://doi.org/10.1186/s13071-021-04701-w

34. Mattah P, Dzorgbe A, Godfred F, Amekudzi LK, Mattah MM, de Souza DK, et al. Diversity in breeding sites and distribution of Anopheles mosquitoes in selected urban areas of southern Ghana. Parasit Vectors. 2017;10:25. D0I 10.1186/s13071-016-1941-3.

35. Bouabida H, Djebbar F \& Soltani N. Etude systématique et écologique des Moustiques (Diptera: Culicidae) dans la région de Tébessa (Algérie). Faun Entomol. 2012;65.19-103.

Page $13 / 19$ 
36. Kudom AA. Larval ecology of Anopheles coluzzii in Cape Coast, Ghana: water quality, nature of habitat and implication for larval control. Malar J. 2015; 14:447. DOI 10.1186/s12936-015-0989-4.

37. Imbahale SS, Paaijmans KP, Mukabana WR, Lammeren RV, Githeko AK, Takken W. A longitudinal study on Anopheles mosquito larval abundance in distinct geographical and environmental settings in western Kenya. Malar J. 2011.10:81. http://www.malariajournal.com/content/10/1/81

38. Ajayi MB, Adeleke MA, Idowu ET, Awolola TS. Surveillance of mosquitoes vectors in Ajumoni Estate Ogun State, Nigeria. An Biol Res. 2010;1(4):16-19 http://scholarsresearchlibrary.com/archive.html

39. Gimnig JE, Ombok M, Kamau L, Hawley WA. Characteristics of Larval Anopheline (Diptera: Culicidae) Habitats in Western Kenya. J Med Entomol. 2001;38(2): 282Đ288.

40. Fournet $F$, Kassié D, Dabiré RK and Salem G. Analyse de la distribution socio-spatiale du paludisme dans une ville moyenne ouest africaine, Bobo-Dioulasso (Burkina Faso). J Int Geosci Environ. 2015;p.146-165. https://doi.org/10.4000/dynenviron.1004

41. Antonio-Nkondjio C, Tene Fossog B, Ndo C, Menze DB, Zebaze TS, Awono-Ambene P, et al. Anopheles gambiae distribution and insecticide resistance in the cities of Douala and Yaoundé (Cameroon): influence of urban agriculture and pollution. Malar $\mathrm{J}$. 2011;10:154 http://www.malariajournal.com/content/10/1/154

42. Sy O, Konaté L, Ndiaye A, Dia I, Diallo A, Taïrou F, et al. Identification des gîtes larvaires d'anophèles dans les foyers résiduels de faible transmission du paludisme « hotspots » au centre-ouest du Sénégal. Bul Soc Pathol Exot. 2016;109:31-38. DOI : 10.1007/s13149-016-0469-2.

43. Mala AO, Irungu LW, Shililu JI, Muturi EJ, Mbogo CM, Njagi JK, et al. Plasmodium falciparum transmission and aridity: a Kenyan experience from the dry lands of Baringo and its implications for Anopheles arabiensis control. Malar J. 2011;10:121 http://www.malariajournal.com/content/10/1/121

44. Benhissen S, Habbachi W, Rebbas Kf, Masna F. Études entomologique et typologique des gîtes larvaires des moustiques (Diptera : Culicidae) dans la région de Bousaâda (Algérie). Bul Soc Roy Sci Liège. 2018;Vol. 87, article, p. 112 - 120.

45. Onchuru TO, Ajamma YU, Burugu M, Kaltenpoth M, Masiga D \& Villinger J. Chemical parameters and bacterial communities associated with larval habitats of Anopheles, Culex and Aedes mosquitoes (Diptera: Culicidae) in western Kenya. Int J Trop Insect Sci. 2016;volume 36, pages146-160.

46. Muturi EJ, Mwangangi J, Shililu J, Jacob BG, Mbogo C, Githure J, et al. Environmental factors associated with the distribution of Anopheles arabiensis and Culex quinquefasciatus in a rice agro-ecosystem in Mwea, Kenya. J Vector Ecol. 2008;33(1):56-63.

47. Mbida Mbida A, Etang J, Akono-Ntonga P, Eboumbou MC, Awono-Ambene P, Tagne D, et al. Nouvel aperçu sur l'écologie larvaire d'Anopheles coluzzii Coetzee et Wilkerson, 2013 dans l'estuaire du Wouri, Littoral-Cameroun. Bull Soc Pathol Exot. 2017;110:92101. DOI 10.1007/s13149-016-0519-9

48. McLaughlin K, Burkot TR, Oscar J, Beebe NW, Russell TL. Defining the larval habitat: abiotic and biotic parameters associated with Anopheles farauti productivity. Malar J. 2019;18:416. https://doi.org/10.1186/s12936-019-3049-7

49. Nilsson LKJ, Sharma A, Bhatnagar RK, Bertilsson S and Terenius O. Presence of Aedes and Anopheles mosquito larvae is correlated to bacteria found in domestic water-storage containers. FEMS Microbiol Ecol. 2018;94. doi: 10.1093/femsec/fiy058.

50. Dada N, Jumas-Bilak E, Manguin S et al. Comparative assessment of the bacterial communities associated with Aedes aegypti larvae and water from domestic water storage containers. Parasit Vectors. 2014;7:391.

\section{Figures}




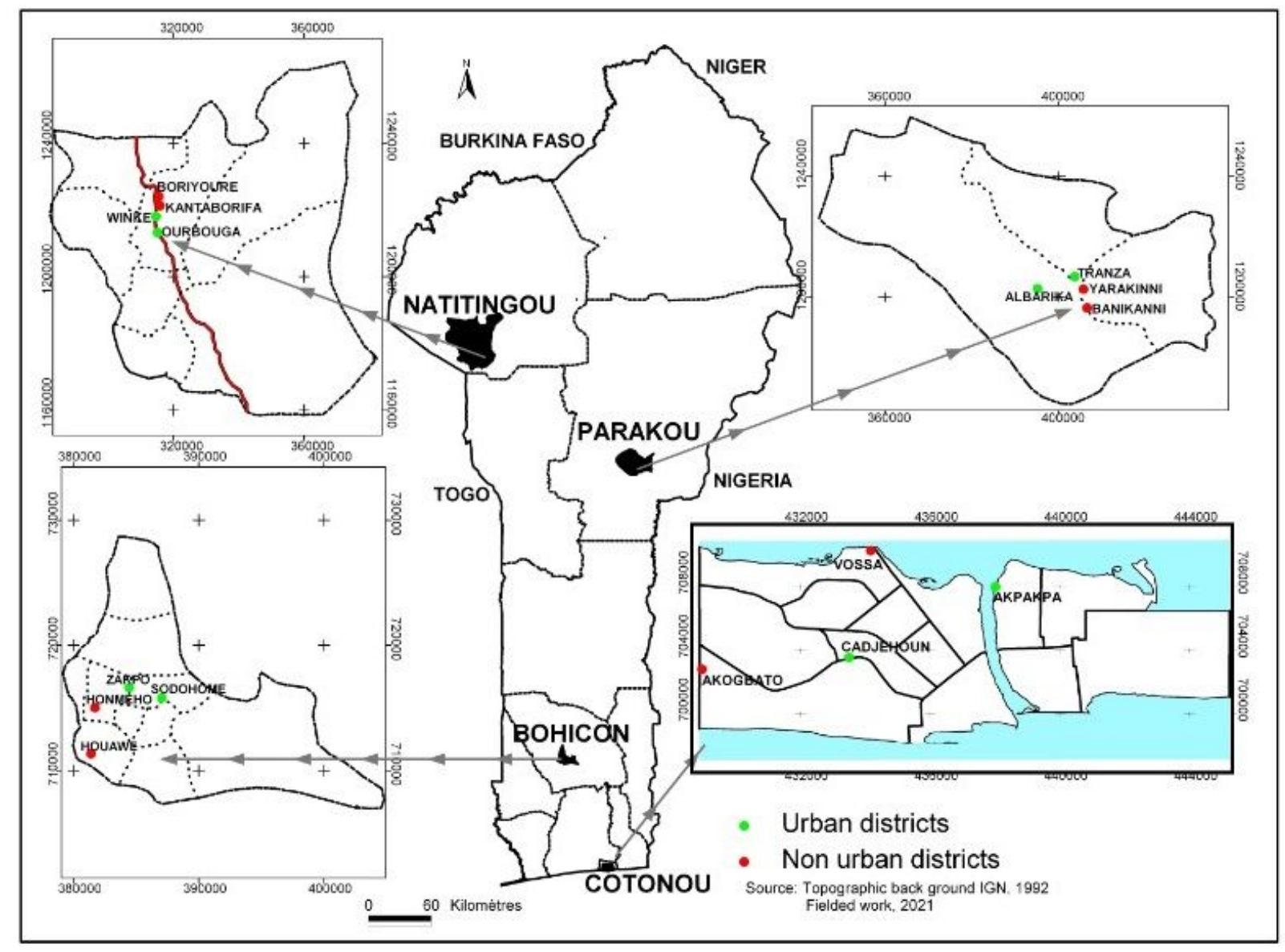

Figure 1

Map of Benin showing the different cities and districts surveyed 


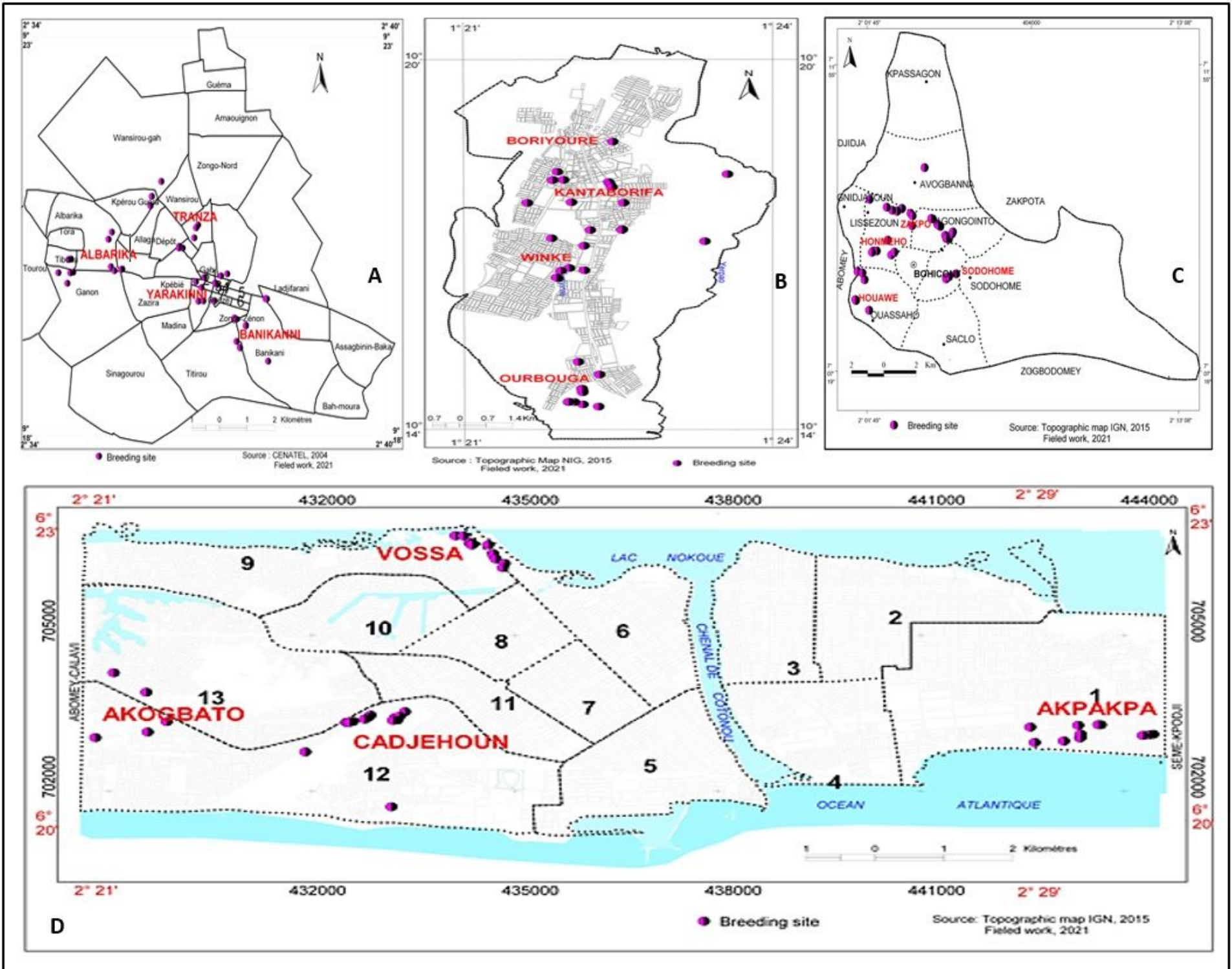

Figure 2

Spatial distribution of the breeding sites prospected in different cities in rainy season (A-Parakou B- Natitingou C- Bohicon D- Cotonou) 


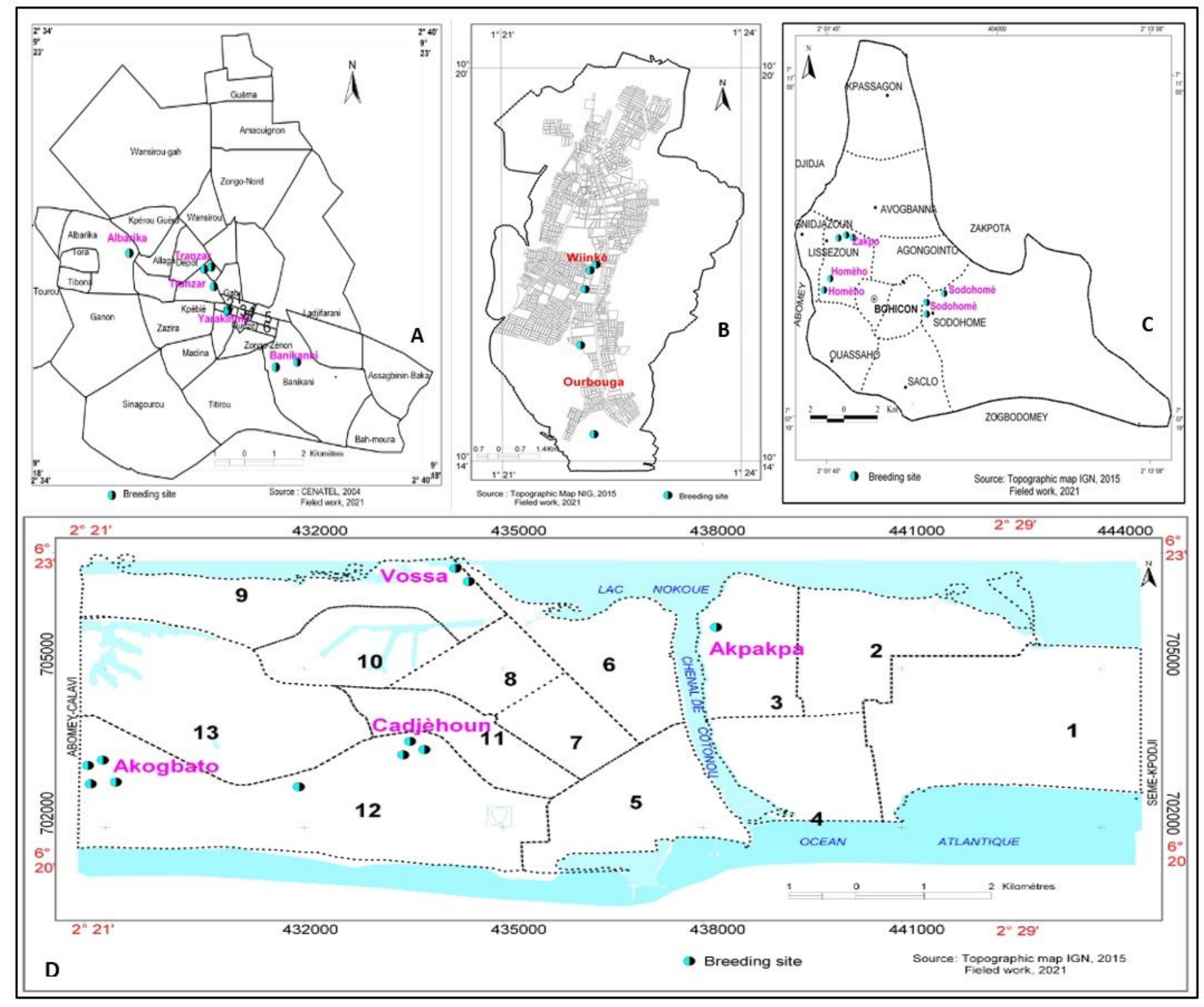

Figure 3

Spatial distribution of the breeding sites prospected in different cities in dry season (A-Parakou B- Natitingou C- Bohicon D- Cotonou) 


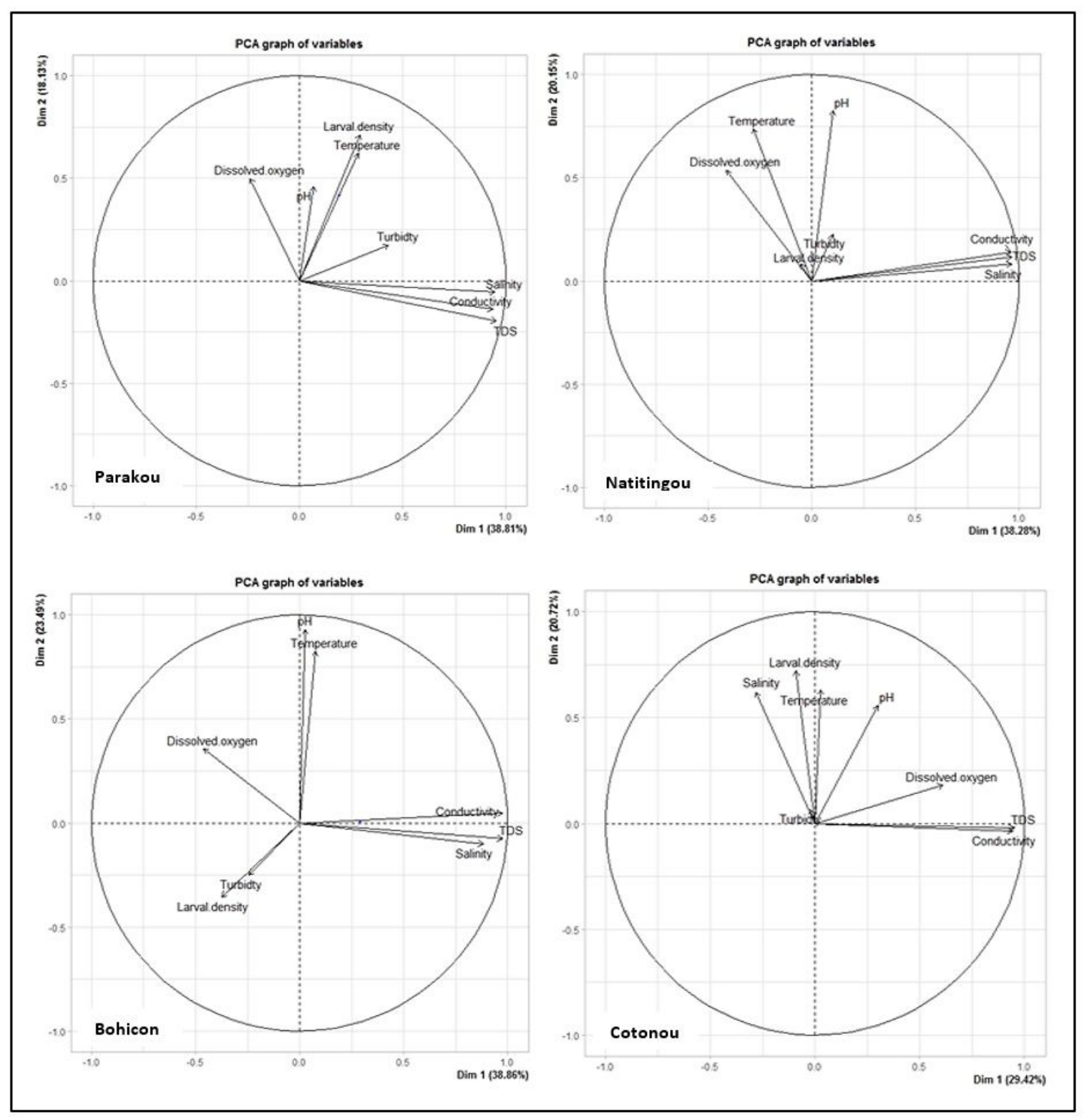

Figure 4

Projection of physicochemical parameters and larval density of Anopheles in the same plane (Dim1 x Dim2 of the PCA) 


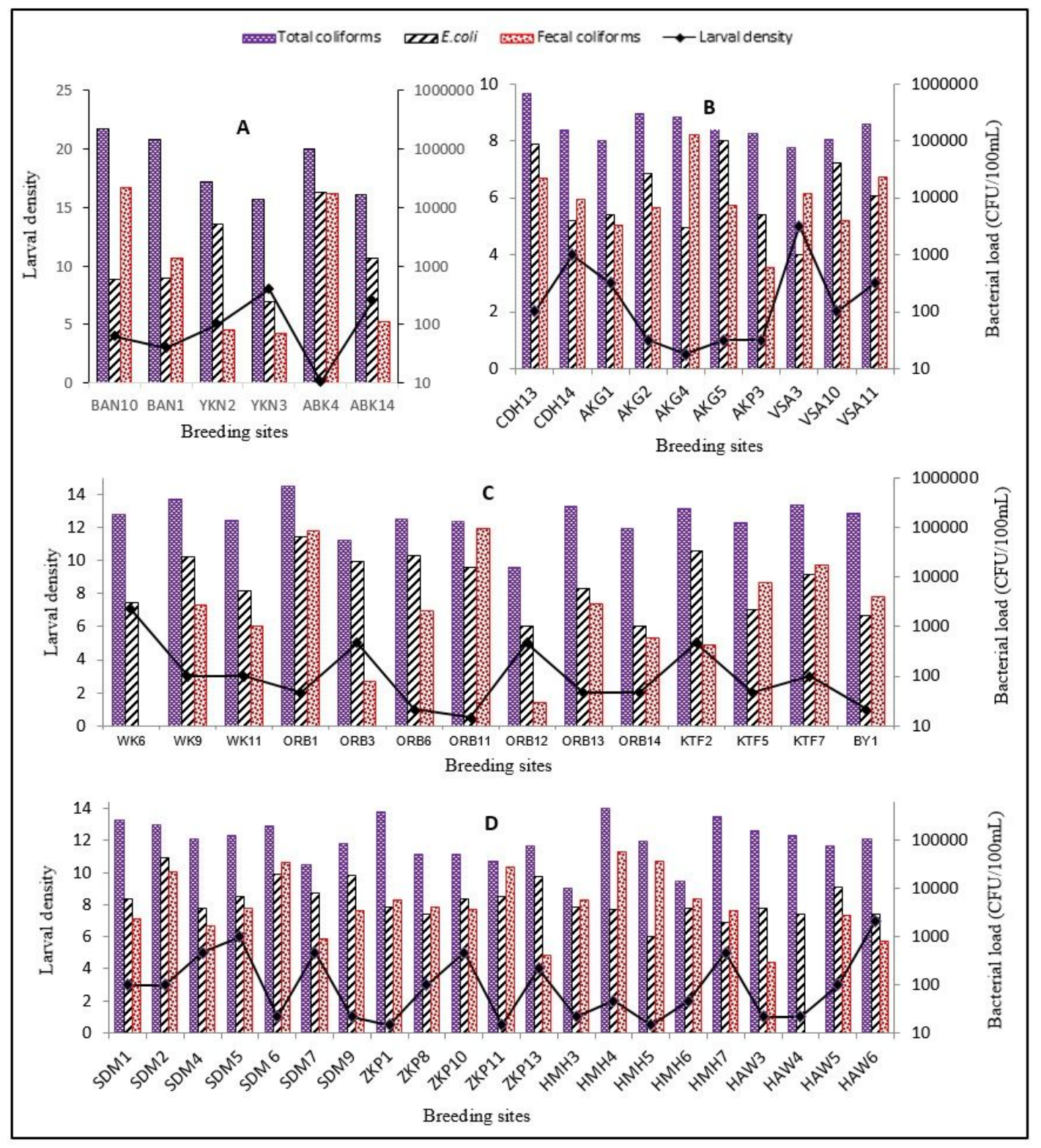

Figure 5

Correlation of the microbiological parameters with the larval density of different breeding sites (A-Parakou B- Natitingou C- Bohicon DCotonou) 